\title{
Voronoi Based Coverage Control with Anisotropic Sensors
}

\author{
Azwirman Gusrialdi, Sandra Hirche, Takeshi Hatanaka and Masayuki Fujita
}

\begin{abstract}
In this paper the coverage control problem for mobile sensor networks is studied. The novelty is to consider an anisotropic sensor model where the performance of the sensor depends not only on the distance but also on the orientation to the target. By adapting the Lloyd algorithm and assuming a fixed and equal sensor orientation, a distributed control law is derived. Aside from coverage, the control law also guarantees collision avoidance between the agents. A simulation is provided to illustrate the results obtained in this paper. Furthermore, a numerical performance analysis to compare the anisotropic sensors modelling to isotropic approximations is performed.
\end{abstract}

\section{INTRODUCTION}

Stimulated by the technological advances and the development of relatively inexpensive communication, computation, and sensing devices, the interest in the research area of coordinated networked control has majorly increased over the past years. One example is the deployment of autonomous vehicles to perform challenging tasks such as search and recovery operations, manipulation in hazardous environments, surveillance and also environmental monitoring for pollution detection and estimation. Deploying multiple agents to perform tasks is advantageous compared to the single agent case: It provides robustness to agent failure and allows to handle more complex tasks.

In this paper, we consider a mobile sensing network of vehicles equipped with sensors to sample the environment. The goal is to drive the sensors/agents to the position such that a given region is optimally covered by the sensors.

Some relevant works on the coverage control problem are [1]-[6]. In [1] the agents move to the optimal configuration which minimizes an objective function. The approach is based on Voronoi tessellation and the Lloyd algorithm. The same problem is considered in [2] with a more realistic model by introducing "limited-range interactions" of the sensors, i.e the sensing range is restricted to a bounded region. The advantage of the Voronoi approach is that the control law is distributed by its nature. Alternative approaches are introduced in [3], [4]. In [3] the authors consider a probabilistic sensing model and a density function to represent the frequency of random events taking place over the mission space. The authors develop an optimization problem that aims at maximizing coverage using sensors with limited ranges, while minimizing communication cost. A potentialfield-based approach to deployment problem in an unknown environment is presented in [4]. Moreover, dynamic coverage

Azwirman Gusrialdi, Takeshi Hatanaka and Masayuki Fujita are with Department of Mechanical and Control Engineering, Tokyo Institute of Technology, Japan fujita@ctrl.titech.ac.jp

Sandra Hirche is with Institute of Automatic Control Engineering, Technische Universität of München, München, Germany hirche@tum. de is considered in [5]. Here, the agents move such that every point in a given area is sensed with a pre-specified coverage level $C^{*}$. The same problem is addressed in [6] under some practical assumptions such as bounded sensing and actuation capacities of the vehicles. However, in the works mentioned above, only a uniform (isotropic) sensor model is considered. In this paper, in contrast to the above papers, we consider the coverage problem with an anisotropic sensor model. This model is more realistic since most of the sensors such as cameras, directional microphones, radars etc are anisotropic. In this paper, one of the main objectives is to investigate the applicability of the Voronoi based approach in [1] to tackle the coverage problem with anisotropic sensor model. For this reason, as a first approach we assume a specific class of anisotropic sensors with elliptic sensing performance level sets as one way of achieving a possibly better approximation to sensor characteristics instead of circles as for the isotropic case. The consideration of a general anisotropic sensor model results in an anisotropic Voronoi tesselation which leads to non-distributed control law, as we will discuss later. However, by assuming fixed, equal sensor orientation, the optimal control law is shown to be decentralized. The idea of deriving the control law for the considered anisotropic sensor model is to transform the anisotropic problem to the isotropic one. By the transformation properties the control law obtained for the isotropic problem also solves the problem for the considered anisotropic case. Simulations are performed in order to validate the proposed approach and for comparison to the approach with isotropic sensor models as over-/underapproximation to truely anisotropic sensors. Numerical performance analysis then indicates a design trade-off between final costs and convergence speed w.r.t. the number of agents. This paper is organized as follows: The problem formulation for the anisotropic sensor model is presented in section 2 . The anisotropic Voronoi partition which is the extension of the ordinary Voronoi partition in Lloyd algorithms and the optimal location of the mobile sensors are derived in section 3. In section 4 the control law for the deployment is derived and collision avoidance is investigated. Numerical performance analysis are provided in section 5 .

\section{PROBLEM Formulation}

Let $Q$ be a convex polytope in $\mathscr{R}^{2}$ including its interior. $\phi(\cdot): Q \rightarrow \mathscr{R}_{+}$is a continuous distribution density function which represents the probability that some event takes place in $Q$. Let $P=\left(p_{1}, \ldots, p_{n}\right)$ be the location of the $n$ identical mobile sensors moving in the region $Q$. Let $\Theta=\left(\theta_{1}, \ldots, \theta_{n}\right)$ be the orientation/attitude of $n$ sensors. The non-decreasing differentiable function $f(\cdot): \mathscr{R}_{+} \rightarrow \mathscr{R}_{+}$indicates the quality 
(a)

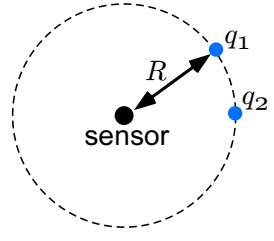

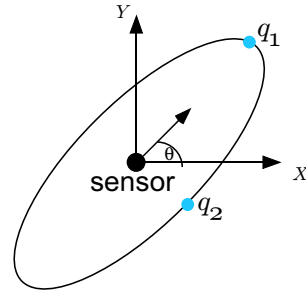

(b)
Fig. 1. (a) Isotropic sensor model, (b) Anisotropic sensor model

of the sensing performance of the sensor, i.e the probability of sensing an event in $Q$. Cortes et. al [1] consider an isotropic sensor with the sensing performance defined as $f\left(\left\|q-p_{i}\right\|\right)$ that degrades with the distance between a point $q \in Q$ and the $i$-th sensor position $p_{i}$. The points where the sensing performance (or probability of sensing) is equal are represented by a circle of radius $R$, and the center is the sensor location. As shown in Fig. 1(a), points $q_{1}$ and $q_{2}$ with the same distance to the sensor will result in the same sensing probability.

In this paper, anisotropic sensors are considered where the degradation of the sensing performance is also affected by the orientation of the sensor w.r.t the point to be sensed. The anisotropic sensor model in this paper is considered by a non-Euclidean distance measure as follows.

Assumption 1: The sensing performance of the anisotropic sensor model is given by the non-Euclidean distance measure $\left\|q-p_{i}\right\|_{L_{i}}$ defined as

$$
\left\|q-p_{i}\right\|_{L_{i}}^{2}=\left(q-p_{i}\right)^{T} L_{i}\left(q-p_{i}\right),
$$

where the matrix $L_{i}$ is positive definite and can be decomposed as $L_{i}=F_{i}^{T} F_{i}$ with

$$
F_{i}=\left[\left(\begin{array}{cc}
\frac{c}{a} & 0 \\
0 & \frac{c}{b}
\end{array}\right)\left(\begin{array}{cc}
\cos \theta_{i} & \sin \theta_{i} \\
-\sin \theta_{i} & \cos \theta_{i}
\end{array}\right)\right]
$$

where $\theta_{i}$ is the orientation of the $i$-th sensor, and $a, b, c>0$ are the parameters.

Observe that the matrix $F_{i}$ is invertible. The level sets of sensing performance of the anisotropic sensor are given by ellipses where the center is the sensor location as shown in Fig. 1(b). Here, $\theta_{i}$ is the orientation of the ellipse, $a, b$ represent the length of major and minor axis of the ellipse respectively. $c$ is a scale factor that decide the sensing performance on a certain level set.

The overall sensing cost incurred by all sensors can be formulated as

$$
\mathscr{H}(P, \Theta, \mathscr{W})=\sum_{i=1}^{n} \int_{W_{i}} f\left(\left\|q-p_{i}\right\|_{L_{i}}\right) \phi(q) d q,
$$

where region $W_{i}$ is the dominance region of the $i$-th sensor and $\mathscr{W}=\left(W_{1}, \ldots, W_{n}\right)$. The challenges addressed in this paper are

1) Find the optimal configuration such that

$$
\min _{P, \Theta, \mathscr{W}} \mathscr{H}
$$

2) Find the control law $u_{i}$ that drives the mobile sensors to the optimal configuration given the mobile sensors dynamics

$$
\dot{p}_{i}=u_{i}
$$

Optimal coverage is achieved by minimizing (3) w.r.t (1) sensor location $P$ and orientation $\Theta$ and (2) the assignment of the dominance regions $\mathscr{W}$.

\section{Optimal PARTITION, LocAtion}

\section{A. Anisotropic Voronoi Partitions}

To minimize (3), we introduce the notion of the Voronoi partition. The Voronoi region of a sensor is defined by all points which are "closer" in the sense of the considered distance measure to that sensor than to any other. For the Euclidean distance measure the Voronoi region $V_{i}$ associated with its generator $p_{i}$ is defined as

$$
V_{i}=\left\{q \in Q\|\|-p_{i}\|\leq\| q-p_{j} \|, \forall j \neq i\right\} .
$$

The Voronoi partition $V_{i}^{*}$ of agent- $i$ for the anisotropic case considered in this paper is defined as follows.

\section{Definition 1:}

$$
V_{i}^{*}=\left\{q \in Q\|\| q-p_{i}\left\|_{L_{i}} \leq\right\| q-p_{j} \|_{L_{j}}, \forall j \neq i\right\} .
$$

This anisotropic Voronoi partition is not only determined by the sensors position but also the sensors orientation $\theta_{i}$ as observable from the matrix $L_{i}$. As a result the anisotropic Voronoi tesselation is no longer composed of convex polytopes, but of curved possibly non-convex regions. Fig. 2(a) and Fig. 2(b) depict the examples of isotropic and anisotropic Voronoi partition.

Lemma 3.1: The boundary between two adjacent $V_{i}^{*}$ and $V_{j}^{*}$ as defined in (6) is a quadratic curve.

Proof: Any point $q$ in $V_{i}^{*} \cap V_{j}^{*}$ which is the boundary of the Voronoi partitions of $V_{i}^{*}$ and $V_{j}^{*}$ satisfies $\left\|q-p_{i}\right\|_{L_{i}}=$ $\left\|q-p_{j}\right\|_{L_{j}}$ i.e. $\left(q-p_{i}\right)^{T} L_{i}\left(q-p_{i}\right)=\left(q-p_{j}\right)^{T} L_{j}\left(q-p_{j}\right)$. It is clear that this equation is quadratic in $q$. Therefore any point in $V_{i}^{*} \cap V_{j}^{*}$ lies on a quadratic curve.

The boundary can be represented as

$$
\left[\begin{array}{l}
x \\
y \\
1
\end{array}\right]^{T}\left[\begin{array}{rrr}
A & \frac{1}{2} C & \frac{1}{2} D \\
\frac{1}{2} C & B & \frac{1}{2} E \\
\frac{1}{2} D & \frac{1}{2} E & K
\end{array}\right]\left[\begin{array}{l}
x \\
y \\
1
\end{array}\right]=0,
$$

where $A, B, C, D, E, K$ in (7) can be computed by solving $\left(q-p_{i}\right)^{T} L_{i}\left(q-p_{i}\right)=\left(q-p_{j}\right)^{T} L_{j}\left(q-p_{j}\right)$. Due to the space limitation, only $A, B, C$ are described which will be used later in this paper. The coefficients of (7) are : $q=(x, y), A=b^{2}\left(\cos ^{2} \theta_{i}-\cos ^{2} \theta_{j}\right)+a^{2}\left(\sin ^{2} \theta_{i}-\sin ^{2} \theta_{j}\right)$, $B=a^{2}\left(\cos ^{2} \theta_{i}-\cos ^{2} \theta_{j}\right)+b^{2}\left(\sin ^{2} \theta_{i}-\sin ^{2} \theta_{j}\right), C=\left(a^{2}-\right.$ $\left.b^{2}\right)\left(\sin 2 \theta_{i}-\sin 2 \theta_{j}\right)$ and $D, E \neq 0$.

Another major difference to isotropic Voronoi tesselations is that anistropic tesselations may contain regions without a generator [7], i.e. a Voronoi cell of an anisotropic Voronoi diagram is not necessarily connected. Moreover, the information of all other sensor positions is required to compute the anisotropic Voronoi diagrams. 


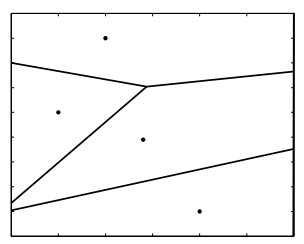

(a)

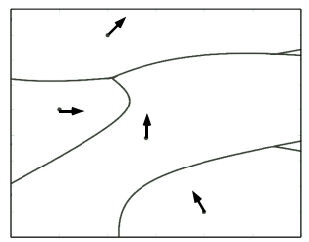

(b)
Fig. 2. (a) Isotropic Voronoi partition, (b) anisotropic Voronoi partition given by (6). As can be seen, the regions on the right side have no generators inside.

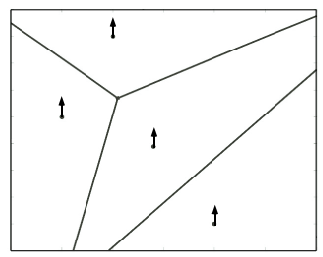

Fig. 3. Anisotropic Voronoi partition with equal orientation

Remark 1: Since the information of all sensor positions is necessary to construct the anisotropic Voronoi diagrams, the resulted control law will be non-distributed. This is in contrast to isotropic Voronoi diagram where only the Delaunay neighbour (agents which have adjacent Voronoi cells) positions are required.

As mentioned above, in general i.e. for the arbitary orientation of the sensors, the Voronoi approach results in non-distributed control law. Therefore, for the remainder of this paper, a more specific case given by the following assumption is considered.

Assumption 2: The orientations of all sensors are equal and fixed over time, i.e. $\theta_{i}(t)=\theta_{j}(t)=\theta, \forall i \neq j$ and $t \geq 0$. This can be achieved by applying a known method (e.g [8]) for making an agreement on the orientation beforehand. This assumption leads to the following lemma.

Lemma 3.2: From assumption 2 and definition 1, the anisotropic Voronoi tessellation is composed of convex polytopes. Moreover, $F_{i}(t)=F_{j}(t)=F$ and $L_{i}(t)=L_{j}(t)=L$ $\forall i \neq j$ and all $t \geq 0$.

Proof: From the assumption 2, $\theta_{i}=\theta_{j}=\theta$, it follows that $A=B=C=0$ in (8) and furthermore $D, E \neq 0$. As a result the boundary of the Voronoi cell is a straight line. Since $Q$ is a convex polytope, the Voronoi tessellation is also composed of convex polytopes. From (2), it is also cleared that $F_{i}=F$ and $L_{i}=L$.

One example of the anisotropic Voronoi diagram with fixed and equal orientations is shown in Fig. 3.

\section{B. Optimal Location}

Corollary 1: The anisotropic Voronoi partition $\mathscr{V}^{*}$ minimizes (3) w.r.t the partition $\mathscr{W}$.

Proof: From definition 1 and since $f$ is a nondecreasing function, it is clear that the Voronoi partition $\mathscr{V}^{*}$ minimizes (3) w.r.t the partition $\mathscr{W}$.

As the orientation is assumed to be fixed and as a result of corollary 1 ,

$$
\min _{P, \Theta, \mathscr{W}} \mathscr{H}=\min _{P} \mathscr{H}_{\mathscr{Y}^{*}}
$$

Assume that the sensing performance $f\left(\left\|q-p_{i}\right\|_{L}\right)=$ $\left\|q-p_{i}\right\|_{L}^{2}$. Then (3) can be written as

$$
\mathscr{H}_{\mathscr{Y} *}(P)=\sum_{i=1}^{n} \int_{V_{i}^{*}}\left\|q-p_{i}\right\|_{L}^{2} \phi(q) d q .
$$

In order to derive the optimal location of the sensors, the above equation can be simplified to

$$
\mathscr{H}_{\mathscr{V}^{*}}(P)=\sum_{i=1}^{n} \int_{V_{i}^{*}}\left\|F\left(q-p_{i}\right)\right\|^{2} \phi(q) d q .
$$

Next, we introduce anisotropic centroidal Voronoi configuration.

Definition 2: Given the set of points $P$ in $Q . C_{V_{i}^{*}}$ is the center of mass (centroid) of an anisotropic Voronoi partition. A Voronoi tessellation is called an anisotropic centroidal Voronoi configuration if

$$
p_{i}=C_{V_{i}^{*}}, \forall i
$$

i.e the points $P$ serve as generators and also centroids for the anisotropic Voronoi tessellations.

The optimal location is given by the following proposition.

Proposition 3.1: The objective function (8) is minimized by the anisotropic centroidal Voronoi configuration .

Proof: Define $\bar{q}, z_{i}$ as $\bar{q}=F q$ and $z_{i}=F p_{i}$ which are points of region and sensors in a space transformed by matrix $F$ called the solution space. Note that the region $Q$ is transformed by $F$ to the convex region $Q_{s}$ and the minimization of (8) in the solution space leads to the minimization in the real physical space. Moreover, from (6) and by applying the transformation matrix $F$, the anisotropic Voronoi partition $V_{i}^{*}$ is transformed to the isotropic Voronoi partition $\left(\bar{V}_{i}\right)$ in the solution space defined as

$$
\bar{V}_{i}=\left\{\bar{q} \in Q_{s} \mid\left\|\bar{q}-z_{i}\right\| \leq\left\|\bar{q}-z_{j}\right\|, \forall j \neq i\right\} .
$$

By applying substitution rule for multiple variables, the integral in (8) can be rewritten as :

$$
\mathscr{H}_{\overline{\mathscr{V}}}(Z)=\sum_{i=1}^{n} \int_{\bar{V}_{i}}\left\|\bar{q}-z_{i}\right\|^{2} \phi(\bar{q})\left|\operatorname{det}\left(F^{-1}\right)\right| d \bar{q} .
$$

with $\mathscr{Z}=\left(z_{1}, \ldots, z_{n}\right)$. Applying the parallel axis theorem, (9) becomes

$$
\mathscr{H}_{\bar{V}}(Z)=\left|\operatorname{det}\left(F^{-1}\right)\right|\left(\sum_{i=1}^{n} J_{\bar{V}_{i}, C_{\bar{V}_{i}}}+\sum_{i=1}^{n} M_{\bar{V}_{i}}\left\|z_{i}-C_{\bar{V}_{i}}\right\|^{2}\right),
$$

where

$$
\begin{gathered}
M_{\bar{V}}=\int_{\bar{V}} \phi(\bar{q}) d \bar{q}, C_{\bar{V}}=M_{\bar{V}}^{-1} \int_{\bar{V}} \bar{q} \phi(\bar{q}) d \bar{q}, \\
J_{\bar{V}, z}=\int_{\bar{V}}\|\bar{q}-z\|^{2} \phi(\bar{q}) d \bar{q} .
\end{gathered}
$$

denote mass, centroid and polar moment of inertia of an anisotropic Voronoi partition respectively. The local mini- 


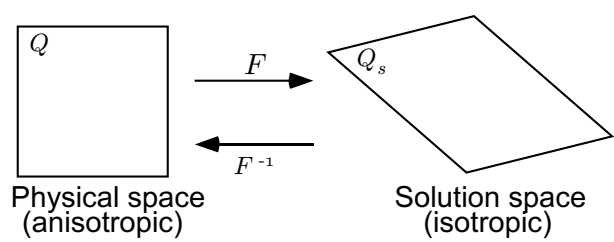

Fig. 4. Transformation between solution and real space

mum is the solution of

$$
\nabla \mathscr{H}_{\bar{V}}=\left[\ldots \frac{\partial \mathscr{H}_{\bar{V}}}{\partial z_{i}} \ldots\right]^{T}=\mathbf{0},
$$

with the partial derivative of (9) given by

$$
\frac{\partial \mathscr{H}_{\bar{V}}}{\partial z_{i}}(Z)=2\left|\operatorname{det}\left(F^{-1}\right)\right| M_{\bar{V}_{i}}\left(z_{i}-C_{\bar{V}_{i}}\right) .
$$

The local minimum points given by $z_{i}=C_{\bar{V}_{i}}$ i.e. the critical points for $\mathscr{H}_{\bar{V}}$ are the centroids of the Voronoi cells in the solution space which are the centroids $C_{V_{i}^{*}}=F^{-1} C_{\bar{V}_{i}}$ of the anisotropic Voronoi partitions.

\section{Continuous Lloyd Descent For Coverage CONTROL}

\section{A. Optimal Control for Fixed Orientation}

In this section, a control law based on Lloyd algorithm to drive the sensors to the location that minimize (3) is derived. The strategy is to transform the control law in the solution space into the real physical space as illustrated in Fig. 4.

Consider the sensors in real space with dynamics given in (4). Set

$$
u_{i}=-k\left(p_{i}-C_{V_{i}^{*}}\right),
$$

where $k$ is a positive gain and $V_{i}^{*}$ is the anisotropic Voronoi partition and assumed to be continuously updated.

Proposition 4.1: By applying the control law in (10), the sensors in the physical space will converge asymptotically to the set of critical points i.e the set of anisotropic centroid Voronoi configurations. If this set is finite, the sensors converge to one of them.

Proof: The dynamics of the sensors in the solution space (isotropic case) is given by

$$
\dot{z}_{i}=\bar{u}_{i}
$$

From [1], it is well-known that the control input given by

$$
\bar{u}_{i}=-k\left(z_{i}-C_{\bar{V}_{i}}\right)
$$

drives the sensors in the solution space to the centroidal Voronoi configuration, the critical points of the objective function (8).

By using the chain rule, the control law in the physical space can be computed by

$$
\begin{aligned}
u & =\dot{p}_{i} . \\
& =\frac{\partial\left(F^{-1} z_{i}\right)}{\partial z_{i}} \dot{z}_{i} . \\
& =-k\left(p_{i}-C_{V_{i}^{*}}\right) .
\end{aligned}
$$

Consider $\mathscr{H}_{Y^{*}}$ as a Lyapunov function. Under the control law (10), $\frac{d}{d t} \mathscr{H}_{V^{*}} \leq 0$. By LaSalle's Principle, the sensors

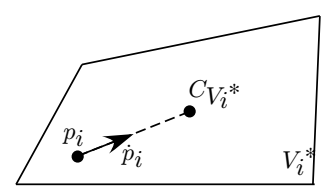

Fig. 5. By the proposed control law, each sensor moves towards the centroid.

converge to the largest invariant set which is the set of anisotropic centroid Voronoi configurations. If this set consists of finite points, then the sensors converge to one of them (see Corollary 1.2 in [1]).

Remark 2: This control law is distributed since each sensor only needs the information of it's neighbour's position to compute the control as observable from (10). Taking out assumption 2 i.e. the case of fixed, unequal orientation will lead to the same control law. However in this case the control law will be non-distributed.

Remark 3: The control law is optimal under the constraint of the fixed orientation. By considering the orientation as optimization variable (non-fixed orientation) as in the original problem will lead to a better result i.e. lower values of $\mathscr{H}$ are achieved.

\section{B. Collision Avoidance Guaranty}

Another advantage of the Voronoi approach is the implicit collision avoidance.

Proposition 4.2: With the control law (10), if there is no collision at $t^{*}$, there will be no collision at $t>t^{*}$.

Proof: The sensors applying the control law (10) will move towards the centroid of its Voronoi cell as shown in Fig. 5. From Lemma 3.2 and the continuity of $\phi(\cdot)$, the centroid is always inside the Voronoi cell and since the Voronoi tessellations are nonoverlapping by construction, no two sensors will come to the same point i.e there will be no collision between the sensors for all $t \geq t^{*}$ if there was no collision at time $t^{*}$.

\section{Numerical Performance Analysis}

The complexity of the control law which is strongly related to the Voronoi tessellation make the analytical performance analysis is difficult to perform. For this reason, here numerical performance analysis is considered. In this section, two kinds of simulations are presented. In a first simulation the proposed approach is validated. Furthermore we compare the using of anisotropic models for design versus isotropic model approximation in a design task.

\section{A. Convergence with Anisotropic Sensors}

First we illustrate the results above through simulation. Assume that there are 4 mobile sensors which sensor parameters $a, b, c, \theta$ are equal to $3,1,1,-\pi / 2$, respectively. The region $Q$ is a rectangle region of $5 \times 4$ unit length. Density function $\phi(q)=1, \forall q$. Assume that at the initial time, $p_{i} \neq p_{j}, \forall i \neq j$ where $p_{i}=\left(x_{i}, y_{i}\right)$ i.e no collision occurs. The results of applying the control law (10) are shown in Fig. 6. Figure 6(a) and Fig. 6(b) show trajectories of the sensors in the transformed and the real physical space respectively. The decreasing of the objective function can be observed from 


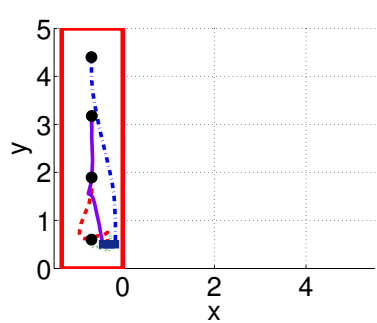

(a)

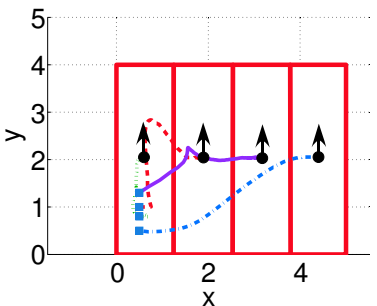

(b)

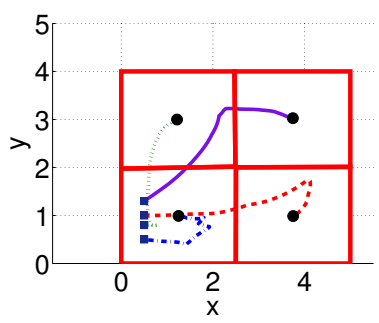

(c)

Fig. 6. Trajectories of the sensors in (a) solution space, (b) real physical space. The square and circle signs show the initial and final position of the agents respectively, (c) the trajectories of the mobile sensors with isotropic sensor model.

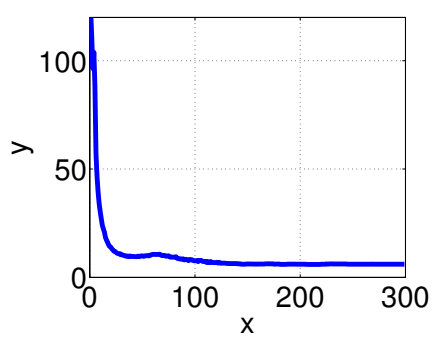

Fig. 7. Cost (objective function) for anisotropic sensor in the real space.

Fig. 7. For comparison, the final configuration of the sensors with isotropic sensor model is depicted in Fig. 6(c).

\section{B. Anisotropic vs. Isotropic Sensor Model Approximation}

In the following we compare the anisotropic sensor modelling vs. using isotropic approximations of the true anisotropic sensors. An important design question is how many agents are necessary to achieve a desired control goal and what is the influence of the agent number on other performance criteria. In the following we will investigate exactly this question under different modelling assumptions for the true anistropic sensor considered in this paper.

1) Simulation goal and method: Assume that anisotropic sensors are used to sense a given region. The goal is to achieve a certain performance in terms of the final cost function value (the cost of the objective function when the agents converge to the critical points). The design task is to choose the necessary number of agents to achieve this goal. Therefore we will compare the cases where we use anisotropic model of the sensor and two other cases where the anisotropic sensor is approximated by an isotropic sensor model. Here we consider two ways to approximate the sensor by using isotropic model, either under-approximate it or over-approximate the sensor as shown in Fig. 8. From simulations with the corresponding sensor models we can derive the necessary number of agents to achieve the desired final cost function value. With these numbers we then investigate the behavior of the "real" system with the anisotropic sensor.

2) Simulation setting: The parameters of the anisotropic sensors $a, b, c, \theta$ are equal to $2,1,1,-\pi / 6$, respectively. The region $Q$ is a square region of side length $l=10$ unit length with $\phi(q)=1, \forall q$. The number of agents are varied from 5 to 60 agents and the number of iterations are 500 steps. The simulation is run once for each number of agent. Initial position are chosen randomly from a square region of side length 3.5 unit length in the middle of the region $Q$ for the different agent numbers as shown in Fig. 9. For a fixed agent number the same initial position is used in order to compare the different modelling approaches. The simulations are carried out first for the case where the isotropic model is used to (under/over)-approximate the anisotropic sensor and when the proposed anisotropic sensor is applied.

3) Results and discussions: Figure 10 shows the influence of number of agents to the final cost for the isotropic and anisotropic sensor model. As expected, for the same number of agents, the anisotropic sensor model leads to a lower final cost than the under-approximation and higher cost than the over-approximation isotropic model. Now the system designed with under/over-approximation is compared to the real one designed with anisotropic sensor model. The comparison is made for number of agents of each sensor model that achieve the same final cost. Here the final cost is assumed to be 100. From Fig. 10, the number of agents for under-approximation, over-approximation and anisotropic sensor model which final cost equal to 100 are given by 32, 8, 22 agents respectively. The behaviour of the system designed with anisotropic sensor model for those number of agents are shown in Fig. 11. As expected, higher number of agents will result to a lower final cost.

\section{Number of Agents vs. Convergence Speed}

Next, through the similar setting as above the influence of the number of agents to the convergence speed of the system is studied. The settling time is used as a metric of the convergence speed which is defined as the number of steps needed to achieve a value in a range of $10 \%$ of the final cost. The simulation result of anisotropic sensor model for different number of agents is shown in Fig. 12. It has been shown analytically that in the one-dimensional case, the convergence rate of Lloyd algorithm slows down as the number of generators becomes large [9]. Here numerical analysis indicates a similar result for the two-dimensional case and the anisotropic sensor model, i.e. large number of agents leads to a slower convergence speed (the settling time is larger). On the other hand, utilizing a large number of agents will lead to a lower final cost. Hence there exists a trade-off between the final cost and convergence speed (settling time) w.r.t the number of agents in the system. Moreover, it will result in a 


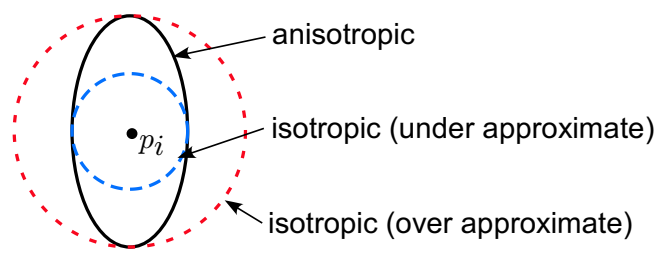

Fig. 8. Approximation of anisotropic sensor with isotropic sensor model.

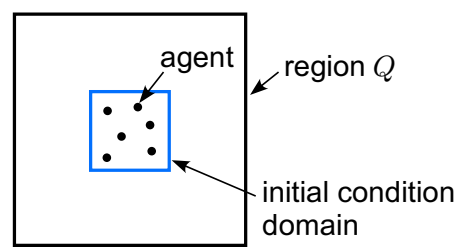

Fig. 9. Example of initial condition for numerical analysis.

higher robustness to agents/sensors failures since for a large number of agents, the final cost is not significanly different (Fig. 10). Note however that, large number of sensors are expensive in terms of the sensors cost.

\section{CONCLUSION AND FUTURE WORKS}

In this paper a first approach for the coverage control with an anisotropic sensor model is presented. The anisotropic sensors considered in this paper are assumed to have elliptic sensing performance level sets. An optimal control law for fixed and equal orientation is derived using a Voronoi based approach with an adapted Lloyd algorithm and a gradient descent approach. The control law is distributed and also guarantees collision avoidance. The efficacy of the proposed control law is confirmed by simulation. The comparison of anisotropic and isotropic sensor model is studied through numerical analysis. It is shown that there is a trade-off between final cost and convergence speed w.r.t number of agents. Currently, the problem with the orientation as optimization variable and a method to make the related control law distributed are investigated. Future work addresses the use of more general anisotropic sensor models and a more suitable alternative approach.

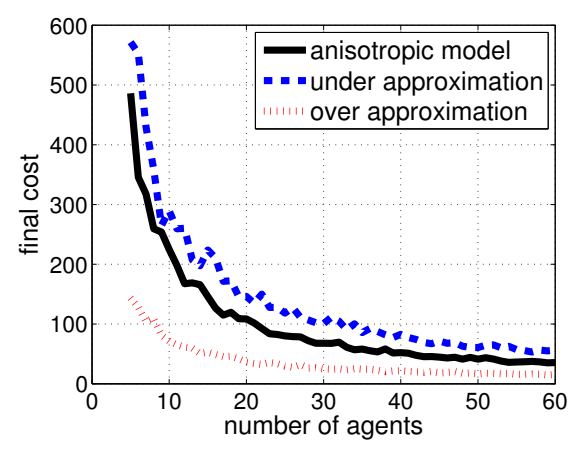

Fig. 10. Number of agents vs. final cost for anisotropic sensor and isotropic sensor model approximation.

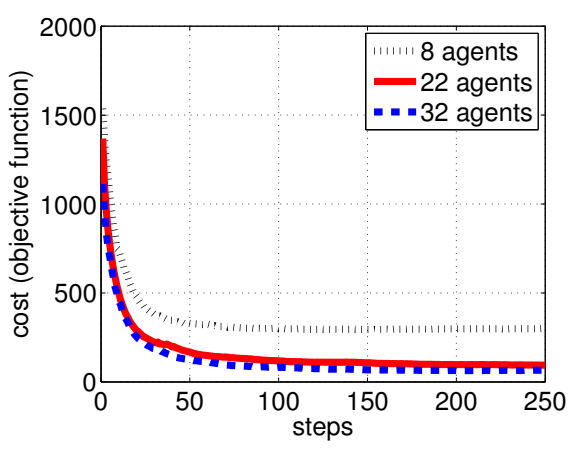

Fig. 11. Cost evolution with anistropic sensors and different agent numbers.

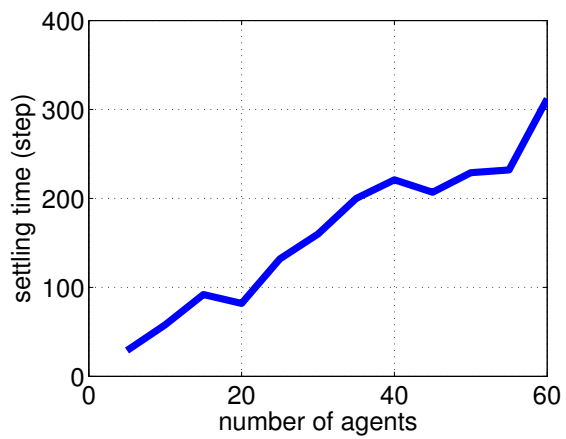

Fig. 12. Number of agents vs. settling time for anisotropic sensor model. Increasing number of agents slows down the convergence speed.

\section{ACKNOWLEDGEMENTS}

This work was supported in part by the Japanese Society for the Promotion of Science (JSPS) in terms of a scholarship granted to the second author.

\section{REFERENCES}

[1] J. Cortes, S. Martinez, T. Karatas, F. Bullo, "Coverage control for mobile sensing networks," IEEE Transactions on Robotics and Automation, Vol. 20, No. 2, pp. 243-255, 2004

[2] J. Cortes, S. Martinez, F. Bullo, "Spatially-distributed coverage optimization and control with limited-range interactions," ESAIM. Control, Optimisation \& Calculus of Variations, Vol. 11, pp. 691-719, 2005

[3] W. Li and C. G. Cassandras, "Distributive cooperative coverage control of sensor networks," Proc. of the IEEE Conference on Decision and Control, pp. 2542-2547, 2005

[4] A. Howard, M. J. Mataric and G. S. Sukhatme, "Mobile sensor network deployment using potential fields : a distributed, scalable solution to the area coverage problem," Proc. of 6th Int. Symposium on Distributed Autonomous Robotics Systems, pp. 299-308, 2002

[5] I. I. Hussein and D. M. Stipanovic, "Effective coverage control for mobile sensor networks," Proc. of the IEEE Conference on Decision and Control, pp. 2747-2753, 2006

[6] P. F. Hokayem, D. M. Stipanovic and M. W. Spong, "Dynamic coverage control with limited communication," Proc. of the American Control Conference, pp. 4878-4883, 2007

[7] F. Labelle, J. R. Shewchuk, "Anisotropic voronoi diagrams and guaranteed-quality anisotropic mesh generation," Proc. of the 19th ACM Symposium on Computational Geometry, pp. 191-200, 2003

[8] Y. Igarashi, M. Fujita and M. W. Spong, "Passivity-based 3D Attitude Coordination: Convergence and Connectivity," Proc. of the IEEE Conference on Decision and Control, pp. 2558-2565, 2007

[9] Q. Du, M. Emelianenko and L. Ju, "Convergence of the Lloyd algorithm for computing centroidal Voronoi tessellations," SIAM Journal on Numerical Analysis, pp. 102-119, 2006 